\title{
LAS RELACIONES SOCIALES Y LAS MOTIVACIONES PARAASISTIR AL FESTIVAL DE MÚSICA DE PERALADA
}

\author{
Josep Ma Prat Forga \\ Departamento de Geografía. Universitat Autònoma de Barcelona \\ josepmaria.prat@uab.cat
}

\section{RESUMEN}

En este trabajo se estudia el perfil de los asistentes al festival de música que se celebra cada verano en Peralada, en la Costa Brava, con el objetivo de comprobar cual es su grado de interés por acudir a dicho evento y por las relaciones sociales que allí se suelen establecer. Para ello se ha realizado una encuesta personal entre los asistentes, confirmándose que la motivación principal es consumir dicho producto musical, aunque un cierto porcentaje de los asistentes también valoran muy positivamente el que antes o después de la función puedan contactar con sus amigos y conocidos, fortaleciendo así sus redes sociales personales.

Palabras clave: relaciones sociales, turismo cultural, festivales de música, motivaciones.

\section{ABSTRACT}

In this workpaper is studied the profile of the assistants to the festival of music that is celebrated in summer in the Costa Brava, with the aim to verify which is his degree of interest to come there and for the social relations that there are in the habit of establishing. For it, there has been realized a personal survey among the assistants, being confirmed that the main motivation is the consumption of this music product, although a significant percentage of them also value very positively that before or after they could contact his friends and acquaintances, strengthening this way his social personal networks.

Key words: social relations, cultural tourism, festivals of music, motivations.

Fecha de recepción: octubre 2012.

Fecha de aceptación: julio 2013. 


\section{INTRODUCCIÓN}

Los cambios producidos en estos últimos años en la demanda turística han provocado un nuevo entorno en el sector, definido por una marcada segmentación del mercado y una gran diversificación de productos, ya que en la actualidad el turista tiene una mayor accesibilidad a la información -especialmente a través de Internet-, es más exigente y reclama una oferta de calidad y diversificada, esperando que las expectativas puestas en un destino turístico sean satisfechas (García Sánchez y Alburquerque, 2003; Vera y Baños, 2010).

Dentro de esta nueva estrategia de diversificación de la oferta turística, el turismo cultural se configura como un elemento esencial para mejorar la competitividad de los destinos -revalorizando los servicios turísticos allí ofrecidos y posibilitando atraer a nuevos consumidores-, constituyendo por sí mismo una gran oportunidad de potenciación turística para aquellas localidades que ofrezcan eventos culturales atractivos y de calidad, como pueden ser los festivales de música de verano, los cuales constituyen un buen ejemplo de oferta complementaria al turismo tradicional, especialmente, pero no exclusivamente, en los destinos maduros de litoral, llegando a ser considerados, en algunos casos, como el motivo principal del desplazamiento al destino (Shapiro y Varian, 1999; Hall, 2007).

De esta manera, algunos de estos festivales de música han pasado de ser un fenómeno puramente festivo, circunscrito a una población local (o como mucho, comarcal), a convertirse en un fenómeno de gran impacto a nivel social y mediático, de manera que, sin dejar de ser un comportamiento minoritario, su importancia ha crecido paralelamente a la dinámica generada por las relaciones sociales que se generan alrededor de la asistencia a estos eventos veraniegos, tanto para políticos como para empresarios, directivos y, en general, para una población con un cierto estatus social y económico (Tabuenca, 2002).

El motivo no es otro que la asistencia a determinados festivales tiene una percepción de un elemento de causalidad directa con las relaciones sociales allí establecidas, y aunque el establecimiento de esta relación causa-efecto se viene realizando desde hace pocas décadas, a medida que se ha ido aumentando la importancia de dichos estos festivales, la dinámica relacional social allí generada también lo ha hecho (Ruhanen, 2008).

En el presente trabajo, se pretende observar empíricamente este fenómeno en un determinado festival, el de Peralada, utilizando para ello fuentes primarias de comportamiento, como son las encuestas directas, apoyadas por una serie de datos estadísticos cuantitativos. En nuestro caso, se ha utilizado una encuesta directa y personal realizada en el mes de agosto de 2012 a una muestra de los asistentes a algunos de los conciertos ofrecidos por el Festival de Música de Peralada (Girona), con el objetivo de realizar los siguientes análisis: en primer lugar, establecer las características sociales que influyen en la asistencia a dichos festivales. En segundo lugar, analizar las características de las relaciones sociales allí establecidas. En tercer lugar, comprobar si esta práctica supone algún tipo de recompensa para dichos asistentes. En cuarto lugar, confirmar si el interés en acudir a estos festivales tiene alguna relación con la motivación por las relaciones sociales que allí se generan.

Por ello, el artículo se estructura de la siguiente forma: tras esta introducción, en los apartados segundo y tercero, se plantea el contexto del estudio, revisando la literatura previa de interés. A continuación, en el apartado cuarto, se analizan los resultados del caso concreto aquí expuesto, presentando primeramente las hipótesis propuestas y la metodología de 
trabajo utilizada para este estudio y luego los resultados obtenidos. En el quinto capítulo, se desarrollan las conclusiones y en el sexto se detalla la bibliografía referenciada.

\section{EL TURISMO, LOS FESTIVALES DE MÚSICA Y LAS REDES SOCIALES}

El consumo de música es un hábito muy extendido en nuestro país, de manera que en el período 2001-2008 el número de conciertos celebrado en España ha pasado de 72.276 (en el año 2001) a 138.613 (en el año 2008), mientras que el porcentaje de espectadores en dicho período ha crecido en un $51 \%$, con un aumento de la recaudación de un $116 \%$, que ha pasado de casi 85 millones de euros en el 2001 a más de 183 millones en el 2008, según datos extraídos de la Fundación Autor-SGAE y del Ministerio de Cultura (2009).

Análogamente, cabe señalar que en el año 2010, el 64,8\% de la población española solía escuchar música diariamente, el 79,8\% lo hacía al menos una vez a la semana y más del 83,7\% al menos una vez al trimestre y el 84,4\% una vez al año (Ministerio de Cultura, 2011).

Además, según el propio Ministerio de Cultura, cada año el $40 \%$ de la población española asiste a espectáculos culturales en directo, destacando los conciertos de música actual, con tasas del $25,9 \%$, observándose una frecuencia inferior en la asistencia a conciertos de música clásica (solamente el 7,7\%), aunque se trata de un público muy satisfecho con este consumo cultural (con una puntuación media de 8,5 sobre 10). En el caso de Cataluña, según datos facilitados por el Idescat, en el año 2006 el 15,9\% de la población catalana asistía a más de tres conciertos de música al año, normalmente en pareja o con amigos.

Esta necesidad cultural de la población ha propiciado la creación de un nuevo producto turístico: los festivales de música. De esta manera, repartidos por toda la geografía española, se celebran al año más de 400 festivales de reconocido interés cultural y artístico, que registran importantes índices de asistencia y que acogen una gran disparidad de público, dada la amplitud y diversidad temática de dichos acontecimientos culturales, los cuales, por su propio atractivo o como complemento a la oferta genérica del destino turístico, están en condiciones de dar satisfacción a las expectativas de la demanda turística (Devesa et al., 2009; Romagosa et al., 2011).

En determinados destinos, el prestigio de dichos festivales presenta un gran poder de atracción, convirtiéndolos en importantes acontecimientos periódicos, de alto nivel cultural y artístico, muchas veces en entornos con un patrimonio cultural de interés, y con una variada oferta de espectáculos distintos para dar satisfacción a un público diferente (Cuadrado y Montoro, 2011). Ahora bien, aunque estos festivales suelen proporcionar una oferta cultural interesante, que en el ámbito urbano puede establecerse a lo largo de todo el año, en las zonas turísticas la gran mayoría tienen lugar durante los meses centrales del verano -julio y agosto- concentrando la oferta más importante, lo cual los convierte en un producto bastante estacional.

Para los destinos, dichos festivales aportan valores únicos, no intercambiables con otros destinos competidores, siendo un elemento básico para diferenciar el destino de la competencia, destacando el incremento de los ingresos, la valorización del tiempo dedicado al ocio y el consumo de cultura. Además, los festivales generan visibilidad mediática para el lugar donde se celebran (Barlés et al., 2012) y, aprovechando la excepcionalidad que supone la celebración del propio festival, se reducen los costes de transacción para el turista (Gonzalo, 2005). 
Sin embargo, con la asistencia a los festivales no solamente se consume un tipo concreto de cultura sino que se crea un espacio, que engloba las relaciones culturales, sociales y humanas. Dichas relaciones presentan una serie de regularidades en la articulación del mismo (Innerarity, 2006), mediante un conjunto de variables interrelacionadas de factores, causas y efectos que permitan comprender la conversión del espacio geográfico en social en cada momento o, lo que es lo mismo, como el espacio geográfico se conforma bajo el peso del conjunto de relaciones sociales que comportan una actuación sobre el medio (Innerarity, 2006). Esta articulación espacial es un hecho social, de modo que el elemento conformador de las relaciones sociales lo será también de las relaciones espaciales (Castillo, 2010).

Dichas relaciones derivan de una necesidad innata de las personas, necesidad que suele cambiar a lo largo del ciclo vital de cada uno (Scott, 1991), con importantes diferencias entre los distintos individuos, tanto en la necesidad de relacionarse con los demás como en conseguir determinados objetivos (Wasserman y Faust, 1994), ya que dichas relaciones sociales pueden cumplir varias funciones: obtener información o apoyo, aprender, construir la propia identidad, proporcionar diversión y entretenimiento, etc., de manera que, por lo general, puede decirse que contribuyen al desarrollo y al bienestar individual (Scott, 1991). Por otra parte, como ya hemos comentado, no todas las personas tienen las mismas necesidades relacionales, puesto que difieren en la fuerza de este impulso. Así, quienes se muestran introvertidos manifiestan una necesidad menor de relacionarse y son más autosuficientes, mientras que los sujetos más necesitados de afiliación experimentarán un mayor malestar ante la carencia de relaciones (Scott, 1991).

Por ello, en los destinos turísticos, los festivales musicales de verano también suelen aprovecharse para las relaciones sociales, que se utilizan para estar en contacto con amigos y con «amigos de mis amigos», y también como una forma de hacerse visible, porque así se forman grupos que comparten intereses, necesidades o preocupaciones dentro de las redes sociales (Wasserman y Faust, 1994; Innerarity, 2006; Castillo, 2010).

Una red social se puede definir como un conjunto de nodos (personas u organizaciones) vinculados a través de una relación social de determinado tipo (Wellman, 1999). Se trata de una serie de relaciones formales e informales, materiales o inmateriales, conscientes y aceptadas, de transmisión de recursos entre unos determinados actores (Sorensen, 2004), formando un conjunto de nodos conectados mediante vínculos, y produciéndose un flujo entre ellos, directamente o a través de otros terceros nodos, de manera que cuanto mayor sea el número de vínculos existentes en la red, mayor será su conectividad e integración (Westlund, 1999).

En este nuevo espacio comunitario, se comparten valores y una determinada organización social, con redes de lazos interpersonales que proporcionan sociabilidad, apoyo, información, sentimiento de pertenencia e identidad social (Wellman, 2001), manifestándose nuevas formas de socialización, estilos de vida y organización social (Cardoso, 1998; Krugman, 2010). Sin embargo, este espacio no es único, sino que está formado por las denominadas «carteras de sociabilidad» (Castells, 2001), que son múltiples (debido a la facilidad de entrada y a los bajos costes de oportunidad, la gente suele pertenecer a varias redes a la vez), dinámicas (cambian en función de los intereses propios), flexibles y mantenedoras de las relaciones sociales más débiles.

Ante esta situación, conocer algunas particularidades del perfil de los asistentes a este tipo de festivales permite preparar las estrategias más adecuadas no solamente para diseñar el 
programa del evento y los servicios que le rodean (Chang, 2006) sino también para segmentar el mercado con el objetivo de dirigir dicha estrategia al público más adecuado (Tkaczynski y Rundle-Thiele. 2011).

\section{EL FESTIVAL DE MÚSICA DE PERALADA}

En estas últimas décadas, Cataluña ha consolidado un sector turístico lo suficientemente voluminoso y de calidad para que las necesidades de la demanda estén cubiertas. Sin embargo, la obligación de continuar siendo competitivos en un mercado cada vez más feroz hace que el sector tenga que idear y aplicar nuevas estrategias para no perder demanda (Romagosa et al., 2011).

Una de ellas es el Festival de Música de Peralada, en el Alt Empodà (Girona), que es la comarca gerundense donde se celebran más festivales de música en verano (Benedicto et al., 2009), siendo una de las principales razones su carácter eminentemente turístico, ya que su territorio abarca una parte de la marca turística Costa Brava.

El municipio que alberga este festival, Peralada (antes denominada «Perelada»), es una población de origen ancestral, que ocupa una extensión de $43,6 \mathrm{~km}^{2}$, con una población censada en el año 2011 de 1.846 habitantes (Idescat 2012), lo que representa una densidad de $42,34 \mathrm{hab} . / \mathrm{km}^{2}$. Presenta una privilegiada situación geográfica ya que se encuentra situado muy cerca de Figueres, la capital de la comarca, así como de la población fronteriza de La Junquera y de los núcleos litorales urbanos de Roses, Cadaqués, Llançà, El Port de la Selva y l'Escala. Además, presenta un importante patrimonio histórico y cultural, ya que en su término municipal se encuentra el castillo de Peralada, antigua residencia de los condes del mismo nombre, que fue restaurado a finales del siglo XIX al estilo de los castillos franceses, así como un conjunto monumental urbano de origen medieval, la iglesia y el claustro góticos, una magnífica biblioteca con más de 80.000 volúmenes, museos del vino, del vidrio y cerámica, y un centro de salud.

La importancia turística del territorio se manifiesta en que, a pesar de que, según datos del Idescat (2012) y del Observatori del Turisme de Catalunya (2011), la comarca del Alt Empordà tenía una población censada de 140.428 habitantes en el año 2011 y solamente representaba el 1,86\% de Cataluña, su oferta hotelera era de 14.537 plazas (el 19,62\% de la marca Costa Brava, a la que pertenece, y el 4,98\% de Cataluña), tenía 38.385 plazas de camping (el 36,38\% de la marca Costa Brava y el 13,77\% de Cataluña), 1.184 plazas de turismo rural (el 38,24\% de la marca Costa Brava y el 7,18\% de Cataluña), 37.471 viviendas principales y 60.063 viviendas secundarias, a lo que no son ajenas su propia situación geográfica ni su relativa proximidad a la Región Metropolitana de Barcelona (que se encuentra a menos de dos horas en coche por autopista de peaje) y a la frontera francesa. Esta importancia turística ha ido pareja con la evolución del destino, que ha ido madurando, y que ha provocado la necesidad de buscar nuevos productos que complementen la tradicional oferta turística de litoral del norte de la Costa Brava, sol y playa, actividades deportivas (con un campo de golf) y de ocio (náutica, gastronomía, rutas enológicas, casino).

Por ello, en una clara apuesta por el turismo cultural -en la comarca se celebran anualmente 14 festivales de este tipo-, cada verano, desde el año 1987, se celebra allí el Festival Internacional de Música, conocido como Festival de Peralada, que en el año 2012 ha recibido más de 
40.000 asistentes entre los meses de Julio y Agosto, con un aumento de 10.000 asistentes en el período 2008-2012, lo que representa un crecimiento del 33\% (Benedicto et al., 2009).

Dicho Festival, que ya ha cumplido 26 ediciones, se celebra actualmente en la Plaza del Castillo, en un auditorio al aire libre situado frente a la fachada principal del castillo, a un paso del casino y de un restaurante buffet al aire libre, que permite cenar cómodamente antes o después del espectáculo. El auditorio, de estilo renacentista con capacidad de 1.600 personas y accesibilidad para discapacitados, acoge espectáculos musicales internacionales variados y de calidad contrastada -conciertos sinfónicos, jazz, pop, música de cámara, ballets, recitales, óperas, espectáculos multidisciplinares de gran formato-. Además, en el mismo recinto del castillo también se encuentran el casino, dos hoteles y cuatro restaurantes.

Se trata de un festival cuya importancia ha servido para que reciba varios reconocimientos internacionales y que sea miembro de la Asociación Europea de Festivales (EFA), de la Ópera Europea, de la Asociación Española de Festivales de Música Clásica (FestClásica) y de la Asociación Española de Teatros, Festivales y Temporadas de Ópera XXI. Paralelamente, la Asociación Cultural Castell de Peralada (fundada en 1986) ha creado el Club de Amigos del Festival de Peralada, con una cuota anual (90 €/año y persona) que permite a sus socios disfrutar de una serie actividades especiales y ventajas exclusivas. Además, el Festival también cuenta con el patrocinio de dieciocho empresas, la colaboración de otras ocho empresas y el apoyo institucional de la Generalitat de Catalunya (Catalunya Turisme y el Institut Català de les Industries Culturals), el Ministerio de Educación, Cultura y Deporte, la Universitat Ramon Llull y el Ayuntamiento de Peralada.

Sin embargo, la asistencia a este Festival presenta varios filtros. El primero, evidentemente, es la distancia geográfica. El segundo es el aforo máximo del auditorio. El tercero es el precio de las entradas, que oscilan entre los $30 €$ (en los laterales y en unas pocas funciones) y los $200 €$ (en platea central y en los eventos más renombrados), aunque los socios del Club de Amigos del Festival de Peralada, los suscriptores del diario La Vanguardia y los socios del club TR3SV de TV3 tienen un descuento del $15 \%$.

\section{ANÁLISIS DEL CASO DE PERALADA}

\section{Hipótesis}

En los últimos años, el número de estudios empíricos sobre el efecto de los festivales de música que se realizan en verano en las zonas turísticas españolas ha crecido notablemente, pero no se ha estudiado si esta motivación musical también tiene alguna relación con una motivación claramente social. La hipótesis aquí planteada es comprobar si en el Festival de Música de Peralada se establecen relaciones sociales entre los asistentes, de tal modo que, en ocasiones, esta motivación tiene tanta o más importancia que la puramente musical.

\section{Métodos y datos}

Primeramente, al tratarse del método más utilizado en la investigación de los segmentos de mercado del turismo de eventos (Getz, 2008), se ha diseñado una encuesta personal, con una serie de preguntas genéricas sobre el sexo, edad, situación personal, nivel de estudios y 
situación laboral de los encuestados, Después, se les ha preguntado si han ido al festival de manera individual o acompañados, si acuden habitualmente o esporádicamente a este evento anual, si en estas fechas están de vacaciones de verano o solamente de fin de semana o si han ido expresamente para ver el espectáculo y a su finalización regresarán a su lugar de origen. A continuación, se les ha pedido que diesen una valoración (en una escala ascendente de 1 al 10) sobre su grado de interés en acudir al Festival y, finalmente, se les ha demandado una valoración (también en una escala ascendente de 1 a 10) sobre cual era el grado de motivación que tenían con respecto a los encuentros sociales que allí se suelen establecer antes o después de la función (charlas, copas, cenas, etc.).

La encuesta, que se realizó los días 5, 8, 9, 10 y 11 de agosto del año 2012 por personal del propio grupo de investigación al que está adscrito el autor de este artículo y con la colaboración de personal de la Fundación Adis, se ha realizado a una muestra escogida al azar entre los asistentes a los espectáculos que allí se celebraban en dichas fechas (tanto a la entrada como a la salida). En total han sido 112 encuestas válidas, cuyo detalle estadístico se presenta en el cuadro siguiente (tabla 1).

Tabla 1

ESTADÍSTICA DESCRIPTIVA DE LA MUESTRA

\begin{tabular}{|c|c|c|c|c|c|c|c|}
\hline & & $\mathrm{n}$ & $\%$ & & & $\mathrm{n}$ & $\%$ \\
\hline $\begin{array}{l}\text { total encuestas } \\
\text { realizadas }\end{array}$ & & 112 & $100 \%$ & & & & \\
\hline \multirow[t]{3}{*}{ género } & & & & $\begin{array}{l}\text { situación } \\
\text { profesional } \\
\text { actual }\end{array}$ & & & \\
\hline & hombre & 58 & $51,79 \%$ & & $\begin{array}{l}\text { directivo, } \\
\text { empresario }\end{array}$ & 30 & $26,79 \%$ \\
\hline & mujer & 54 & $48,21 \%$ & & $\begin{array}{l}\text { técnico medio o } \\
\text { superior }\end{array}$ & 10 & $8,93 \%$ \\
\hline \multirow[t]{5}{*}{ grupo de edad } & & & & & funcionario & 15 & $13,39 \%$ \\
\hline & $<25$ & 7 & $6,25 \%$ & & otros trabajos & 21 & $18,75 \%$ \\
\hline & $25-40$ & 26 & $23,21 \%$ & & parado & 3 & $2,68 \%$ \\
\hline & $40-60$ & 57 & $50,89 \%$ & & $\begin{array}{l}\text { jubilado, } \\
\text { discapacitado }\end{array}$ & 10 & $8,93 \%$ \\
\hline & $>60$ & 22 & $19,64 \%$ & & estudiante & 11 & $9,82 \%$ \\
\hline \multirow[t]{3}{*}{ nivel educativo } & & & & & labores del hogar & 12 & $10,71 \%$ \\
\hline & $\begin{array}{l}\text { estudios } \\
\text { primarios }\end{array}$ & 19 & $16,96 \%$ & $\begin{array}{l}\text { estancia } \\
\text { durante } \\
\text { el festival }\end{array}$ & & & \\
\hline & $\begin{array}{l}\text { estudios } \\
\text { medios }\end{array}$ & 44 & $39,29 \%$ & & vacaciones & 81 & $72,32 \%$ \\
\hline
\end{tabular}


Tabla 1 (continuación)

ESTADÍSTICA DESCRIPTIVA DE LAMUESTRA

\begin{tabular}{|c|c|c|c|c|c|c|c|}
\hline & & $\mathrm{n}$ & $\%$ & & & $\mathrm{n}$ & $\%$ \\
\hline & $\begin{array}{l}\text { estudios } \\
\text { superiores }\end{array}$ & 49 & $43,75 \%$ & & fin de semana & 25 & $22,32 \%$ \\
\hline \multirow[t]{5}{*}{$\begin{array}{l}\text { situación } \\
\text { personal actual }\end{array}$} & & & & & $\begin{array}{l}\text { desplazamiento } \\
\text { de ida y vuelta }\end{array}$ & 6 & $5,36 \%$ \\
\hline & $\begin{array}{l}\text { sin pareja } \\
\text { estable }\end{array}$ & 33 & $29,46 \%$ & $\begin{array}{l}\text { forma de } \\
\text { acudir al } \\
\text { festival }\end{array}$ & & & \\
\hline & $\begin{array}{l}\text { con pareja } \\
\text { estable }\end{array}$ & 79 & $70,54 \%$ & & acompañado & 101 & $90,18 \%$ \\
\hline & & & & & individualmente & 11 & $9,82 \%$ \\
\hline & & & & $\begin{array}{l}\text { va al } \\
\text { festival }\end{array}$ & & & \\
\hline \multirow[t]{2}{*}{$\begin{array}{l}\text { relaciones } \\
\text { sociales }\end{array}$} & & & & & habitualmente & 81 & $78,64 \%$ \\
\hline & $\begin{array}{l}\text { nivel } \\
\text { medio de } \\
\text { satisfacción } \\
\text { (sobre 10) }\end{array}$ & 6,12 & & & esporádicamente & 22 & $21,36 \%$ \\
\hline
\end{tabular}

Fuente: elaboración propia con Excel.

Como se aprecia en el cuadro anterior, asistir a alguno de los conciertos del Festival de Peralada es una experiencia común entre hombres y mujeres, sin una diferencia significativa de género, aunque sí que se observan algunas diferencias más significativas en el resto de variables sociodemográficas. Así, el nivel educativo de los asistentes al Festival es elevado; están mayoritariamente entre 40 y 60 años; y disfrutan de una situación profesional estable. Del mismo modo, dichos asistentes suelen tener pareja estable o estar en estos momentos de vacaciones de verano, siendo realizada esta actividad de forma conjunta con familiares o amigos. También, suelen acudir habitualmente en los últimos años.

Según se desprende de la encuesta, la valoración media del grado de motivación que los encuestados manifiestan sobre la posibilidad de mantener allí relaciones sociales es bastante aceptable (un 6,12 sobre 10). En cuanto al grado de interés en acudir al Festival (variable A), la valoración media dada por los encuestados es de 6,79 (sobre 10), con una desviación estándar de 1,21, un valor máximo de 9 y un mínimo de 4, y un nivel de confianza de 0,23 (al 95\%).

A continuación, a partir de las respuestas sobre el grado de motivación manifestado respecto a las relaciones sociales que se establecen con la asistencia al Festival (variable B) se ha obtenido el siguiente cuadro estadístico (tabla 2), donde se aprecia que la valoración media es casi de 6,12 (sobre 10), con una desviación estándar de 1,3, un valor máximo de nueve y un mínimo de tres, y un nivel de confianza de 0,24 (al 95\%). 
Tabla 2

ESTADISTICAS DESCRIPTIVAS DEL INTERÉS EN ACUDIR AL FESTIVAL (A) Y DE LA MOTIVACIÓN CON LAS RELACIONES SOCIALES (B)

\begin{tabular}{|l|c|c|}
\hline & A & B \\
\hline media & 6,7857 & 6,1161 \\
error típico & 0,1145 & 0,1228 \\
mediana & 7 & 6 \\
moda & 7 & 7 \\
desviación estándar & 1,2113 & 1,2997 \\
varianza de la muestra & 1,4672 & 1,6891 \\
curtosis & $-0,367$ & $-0,429$ \\
coeficiente de asimetría & $-0,073$ & $-0,094$ \\
rango & 5 & 6 \\
mínimo & 4 & 3 \\
máximo & 9 & 9 \\
suma & 760 & 685 \\
cuenta & 112 & 112 \\
nivel de confianza $(95,0 \%)$ & 0,2268 & 0,2433 \\
\hline
\end{tabular}

Fuente: elaboración propia con Excel.

Como se puede observar en la tabla superior, ambas variables presentan unos coeficientes de curtosis bajos y negativos, por lo que sus curvas casi presentan una distribución normal y casi simétrica, aunque ligeramente platicúrtica, con una menor concentración de valores alrededor de la media, y sin tener colas pesadas, compensándose esa «baja varianza» con una mayor variabilidad en el resto de la distribución.

A continuación, se ha realizado el análisis de correlación entre ambas variables y, posteriormente, un modelo de regresión lineal múltiple, ya que es un procedimiento estadístico que permite determinar si existe o no relación de dependencia entre dos o más variables, de manera que conociendo los valores de una variable independiente, se puedan estimar los valores de varias variables dependientes (Cohen y Cohen, 1983).

\section{Resultados del análisis}

En primer lugar, a partir de los resultados de la encuesta, se ha creado una matriz de datos y mediante un análisis de correlación lineal entre dos variables aleatorias se ha calculado el coeficiente de correlación lineal de Pearson ( $r$ ), cuyo valor oscila entre -1 y +1 . En nuestro caso, se han comparado las respuestas dadas sobre el interés en acudir al Festival y la motivación con las relaciones sociales allí establecidas, obteniéndose el gráfico siguiente (figura 1), donde, además de la correlación entre ambas variables, aparecen la ecuación de la línea de tendencia y el valor del coeficiente $\mathrm{r}$. 
Como se observa en dicho gráfico, en el caso presente, el coeficiente $r$ presenta un valor de 0,3043, (bastante inferior a la unidad), lo que significa que entre ambas variable existe una correlación lineal positiva débil, ya que, aunque hay una cierta correlación creciente, el valor de $\mathrm{r}$ está más cercano a 0 que a +1 (el valor de $\mathrm{r}$ se aproxima a +1 cuando la correlación tiende a ser lineal directa, y se aproxima a -1 cuando la correlación tiende a ser lineal inversa) (Etxeberria, 1999). Es importante notar que la existencia de una cierta correlación entre ambas variables no implica causalidad.

La valoración media obtenida con respecto al grado de interés en acudir al Festival es mayor que la de la motivación respecto a las relaciones sociales allí establecidas $(6,79$ frente a 6,12). De este modo, entre los asistentes al Festival prima el interés en disfrutar del espectáculo, aunque las relaciones sociales que allí se establecen también tengan interés.

Figura 1

RELACIÓN ENTRE EL INTERÉS EN ACUDIR AL FESTIVAL Y LA MOTIVACIÓN CON LAS RELACIONES SOCIALES ESTABLECIDAS

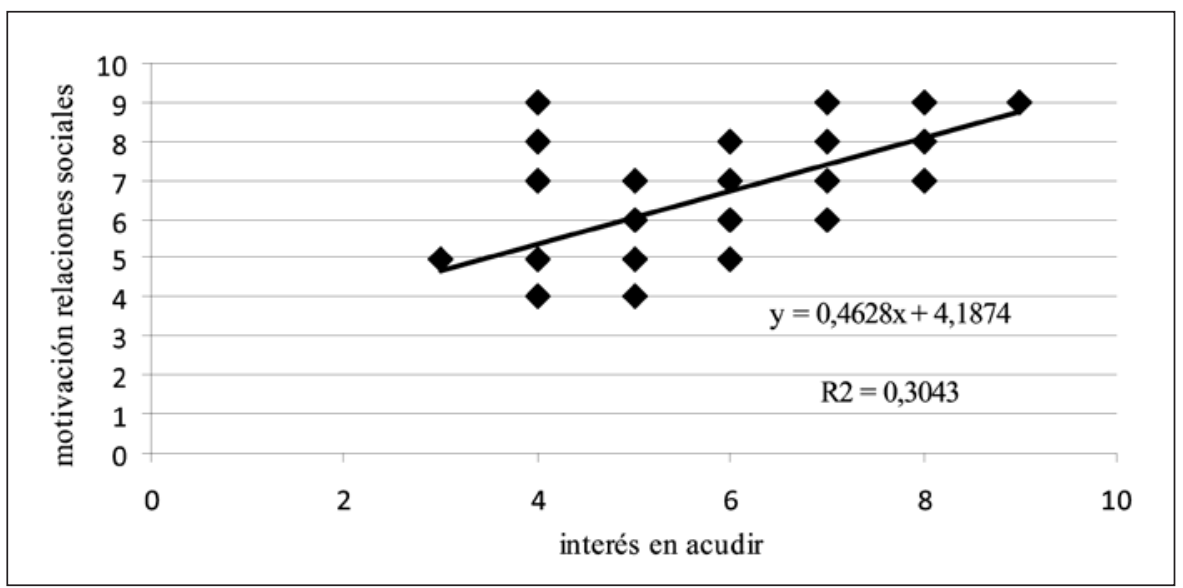

Fuente: elaboración propia con Excel.

Seguidamente, se ha procedido a comprobar el grado de relación entre la motivación con las relaciones sociales establecidas en el Festival (que se toma como variable dependiente) con un conjunto de variables independientes (nivel de formación, situación profesional y si van al festival de forma individual o acompañados), para lo cual se ha calculado su regresión lineal.

A continuación, se ha realizado el mismo procedimiento para comprobar la relación entre la misma variable dependiente con cada una de las siguientes variables: el género, la edad de los asistentes, la situación personal (si viven en pareja o no), la forma de acudir al espectáculo (solos o acompañados) y el tipo de estancia en aquellos momentos (vacaciones, fin de semana, ida y vuelta).

Los resultados obtenidos con Excel para cada uno de estos análisis de regresión son los que se muestran en el cuadro siguiente (tabla 3). 
Tabla 3

RESULTADOS DE LOS CÁLCULOS DE LA REGRESIÓN LINEAL MÚLTIPLE

\begin{tabular}{|l|ccccc|}
\hline variable & $\begin{array}{c}\text { coeficiente de } \\
\text { correlación } \\
\text { múltiple }\end{array}$ & $\begin{array}{c}\text { coeficiente de } \\
\text { determinación } \mathrm{R}^{2}\end{array}$ & $\begin{array}{c}\mathrm{R}^{2} \\
\text { ajustado }\end{array}$ & $\begin{array}{c}\text { error } \\
\text { típico }\end{array}$ & observaciones \\
\hline total encuestados & 0,76980 & 0,59250 & 0,58120 & 0,84110 & 112 \\
\hline hombres & 0,81587 & 0,66564 & 0,62855 & 0,75793 & 58 \\
mujeres & 0,72376 & 0,52382 & 0,49525 & 0,92237 & 54 \\
\hline$<25$ años & 0,98036 & 0,96111 & 0,69167 & 0,35355 & 7 \\
$25-40$ años & 0,72829 & 0,53041 & 0,46637 & 0,71574 & 26 \\
$40-60$ años & 0,73342 & 0,53791 & 0,51175 & 0,83681 & 57 \\
$>60$ años & 0,9075 & 0,82355 & 0,79415 & 0,77299 & 22 \\
\hline sin pareja estable & 0,81691 & 0,66734 & 0,63293 & 0,77143 & 33 \\
con pareja estable & 0,75092 & 0,56388 & 0,54644 & 0,88322 & 79 \\
\hline individualmente & 0,92319 & 0,85228 & 0,78898 & 0,45094 & 11 \\
acompañado & 0,75388 & 0,56834 & $55,50 \%$ & 0,87448 & 101 \\
\hline ida y vuelta & 0,94398 & 0,89111 & 0,72777 & 0,71286 & 6 \\
vacaciones & 0,85805 & 0,73626 & 0,72598 & 0,67636 & 81 \\
fin de semana & 0,53237 & 0,28342 & 0,18105 & 1,07712 & 25 \\
\hline
\end{tabular}

Fuente: elaboración propia con Excel.

El coeficiente de determinación $\left(\mathrm{R}^{2}\right)$ indica el porcentaje del ajuste que se ha conseguido con el modelo lineal (es decir, el porcentaje de la variación de una variable). En el caso presente es la motivación por las relaciones sociales generadas con la asistencia al Festival. Se explica mediante el modelo lineal que aquí se ha estimado (a través del comportamiento de la otra variable). En nuestro caso, es el grado de interés en acudir al Festival. De esta manera, a mayor porcentaje obtenido, mejor es el modelo para predecir el comportamiento de la primera variable. También se puede entender este coeficiente de determinación como el porcentaje de varianza explicada por la recta de regresión, con un valor entre 0 y 1 y siempre igual al cuadrado del coeficiente de correlación (r).

En el caso aquí analizado, el coeficiente de determinación $\mathrm{R}^{2}$, que mide la bondad del ajuste, presenta un valor de 0,5925 , lo que indica que existe una relación media entre ambas variables, ya que el 59,25\% de los asistentes al Festival tienen una motivación adicional con las relaciones sociales allí establecidas, aunque si nos fijamos con mayor grado de detalle en los análisis parciales con cada una de las variables independientes pueden hacerse algunos comentarios.

En efecto, según se desprende de los valores de $\mathrm{R}^{2}$ presentados en la tabla anterior, en primer lugar, en el caso de los varones existe una relación mayor entre la asistencia al festival y el interés en las relaciones sociales que allí se establecen (el 66,56\% de los varones frente al $52,38 \%$ de las mujeres). 
En segundo lugar, los asistentes menores de 25 años y los mayores de 60 años son los que tiene más interés en dichas relaciones. Así, el 96,11\% de los jóvenes tiene interés en las relaciones sociales que allí tienen lugar (en su caso, principalmente, reunirse con otros amigos de su edad), como también ocurre con el 82,35\% de los más mayores. Por contra, solamente el 53\% de los asistentes de edad intermedia (entre 25 y 60 años) manifiestan un interés en las relaciones sociales allí establecidas.

En tercer lugar, el $85,23 \%$ de los que asisten solos al espectáculo presentan interés en dichas relaciones, frente al 56,83\% en el caso de los que acuden allí en compañía; y lo mismo que ocurre con el $66,73 \%$ de los que manifiestan no tener pareja estable en estos momentos, frente al $56,39 \%$ de los que sí que la tienen.

Finalmente, una mayoría (más del 70\% en ambos casos) de los que acuden expresamente para ver el espectáculo y de los que ya se encuentran en aquella zona de vacaciones veraniegas manifiestan interés en las relaciones sociales allí establecidas, frente al 28,34\% en el caso de los que solamente están allí durante el fin de semana.

\section{CONCLUSIONES}

En el actual contexto mundial, los cambios en las demandas de los turistas y la competitividad creciente entre los destinos han provocado la necesidad de ofertar nuevos productos turísticos, más personalizados. Dentro de este nuevo entorno, en los destinos maduros de litoral cada vez cobra más importancia el turismo cultural, y una de sus expresiones son los festivales de música de verano.

En estos eventos aparecen múltiples espacios, cada uno con distinto significado. Por una parte está el espacio puramente cultural, donde se disfruta del espectáculo cultural. Por la otra, el espacio geográfico, es decir, la localidad donde éste se desarrolla. Y luego, el espacio social, donde se establecen las relaciones sociales entre los asistentes.

Según se desprende de los resultados del caso aquí analizado, el Festival de Peralada, el perfil socio-demográfico de los asistentes a este festival es indistintamente de hombres o mujeres, mayoritariamente entre 40 y 60 años, con estudios medio o superiores, con pareja estable en estos momentos y con un trabajo bastante bien remunerado o que al menos presenta una cierta seguridad. Acuden al festival acompañados generalmente por su pareja y suelen ser habituales, año tras año, aprovechando su estancia cercana durante las vacaciones veraniegas.

En su interés por asistir, predomina ligeramente el consumo cultural frente a la motivación por las relaciones sociales, ya que el grado de interés en acudir al Festival es medio-alto (un 6,79 sobre 10), mientras que la motivación relacional es de 6,12 (sobre 10). Aún así, en este festival se crean distintos espacios, geográficos, culturales y sociales, donde los individuos de un determinado estatus social se pueden encontrar periódicamente, compartiendo amistad, información y contactos. De esta manera, las relaciones sociales allí establecidas tienen una cierta importancia para dichos asistentes.

Esta importancia varía en función de diversos parámetros. Así, aunque la etapa dorada de las relaciones de amistad es la adolescencia (Innerarity, 2006), y este aspecto también se confirma en el presente trabajo, y durante la edad adulta y la madurez estas relaciones vuelven a tener cierta importancia, tal como también indican Caradec (2001) y Pirkko (2006), entre 
otros. Se trata de un tipo de relaciones que, de una u otra manera, se encuentran presentes a lo largo de todo el ciclo vital de las personas, aunque con unas características y funciones diferentes en cada momento. A diferencia de las relaciones familiares, las relaciones sociales, especialmente las de amistad, son voluntarias, suelen basarse en la similitud de intereses, experiencias, necesidades y objetivos, y están orientadas hacia la diversión, la satisfacción personal y/o la consecución de un determinado interés. Tampoco puede olvidarse que la situación personal y/o profesional de cada individuo también determina con qué personas se producen las relaciones y cual es la finalidad que se pretende alcanzar.

En este trabajo también se ha constatado que casi el $60 \%$ de los asistentes al Festival de Peralada tienen una motivación adicional con dichas relaciones. Sin embargo, este porcentaje varía significativamente en función de la edad, situación personal y situación geográfica de cada uno. Sea como sea, estas relaciones constatan la cada vez mayor importancia de las redes sociales para conseguir determinados fines.

En consecuencia, la hipótesis aquí planteada (comprobar si en el Festival de Música de Peralada se establecen relaciones sociales entre los asistentes, de tal modo que, en ocasiones, esta motivación tiene tanta o más importancia que la puramente musical) no se cumple totalmente, ya que predomina el aspecto lúdico, aunque las relaciones tampoco son desdeñables.

Ahora bien, tras las conclusiones aquí presentadas, hay que tener en cuenta algunas reflexiones. En primer lugar, la muestra no es totalmente significativa, ya que no cubre los asistentes a todos los diferentes tipos de espectáculos ofrecidos a lo largo del Festival. En segundo lugar, el trabajo se ha focalizado en un festival de prestigio internacional que presenta unas claras barreras de entrada (el medio de desplazamiento, el aforo del local y los precios de las entradas, por ejemplo). En tercer lugar, el tamaño de la muestra, de forma que a pesar de haberse realizado 112 encuestas, algunos de los análisis adolecen de un número significativo de observaciones.

Por ello, aunque las conclusiones aquí establecidas solamente pueden aplicarse a este festival, quizá también podrían extrapolarse a algún otro caso muy similar, es decir, a festivales de música de reconocido prestigio que se realicen en el verano en destinos de litoral donde el estatus social tenga una cierta importancia (como, por ejemplo, en el caso de la misma Costa Brava, el Festival de Cap Roig, en Calella de Palafrugell). Por ello, en el futuro sería interesante ampliar el abanico de la muestra, tanto con más entrevistados, de todo tipo y condición, como en distintos festivales, lo que seguramente serviría para realizar comparaciones y confirmar si las conclusiones obtenidas para este festival pueden generalizarse al reto de festivales del mismo tipo.

\section{BIBLIOGRAFÍA}

BARLÉS, M.J., ABELLA, S. y MUR, M. (2012): «Perfil del turista de festivales: el caso del Festival Internacional de las Culturas Pirineos Sur». Cuadernos de Turismo, $\mathrm{n}^{\circ}$ 30, 63-90. BENEDICTO, S., MORERA, S. y VERDÚ, M. (2009): Gestió i impacte dels festivals de Música d'estiu de la província de Girona. Girona, Diputación de Girona, 167 pp.

CARADEC, V. (2001): Sociologie de la vieillesse et du vieillissement. París, Nathan, 128 pp.

CARDOSO, C. (1998): Para una sociología do ciberespaço. Comunidades virtuais em portugues. Oeiras, Celta Editora. 
CASTELLS, M. (2001): La Galaxia Internet. Reflexiones sobre Internet, empresa y sociedad. Barcelona, Areté.

CASTILlO, A. (2010): Introducción a las Relaciones Públicas. Málaga, Instituto de Investigación en Relaciones Públicas.

CHANG, J. (2006): «Segmenting tourist to aboriginal cultural festivals: An example in the Rukai tribal area, Taiwan». Tourism Management, $\mathrm{n}^{\circ}$ 27, 1224-1234.

COHEN, J. y COHEN, P. (1983): Applied multiple regression/correlation analysis for the behavioral sciences. Hillsdale, NJ, Lawrence Erlbaum Associates.

CUADRADO, M. y MONTORO, J. (2011): «Live and prerecorded popular music consumption». Journal of Cultural Economics, $n^{\circ} 35$ (1), 19-48.

DEVESA, M., HERRERO, L.C. y SANZ, J.A. (2009): «Análisis económico de la demanda de un festival cultural». Estudios de Economía Aplicada, no 27 (1), 137-138.

ETXEBERRIA, J. (1999): Regresión Múltiple. Cuadernos de Estadística.Salamanca, Ediciones La Muralla Espérides.

FUNDACIÓN AUTOR-SGAE y MINISTERIO DE CULTURA (2009): «Anuario SGAE de las artes escénicas, musicales y audiovisuales 2007-2009». Disponible en http://www. artenetsgae.com/anuario/home.html

GARCÍA SÁNCHEZ, A. y ALBURQUERQUE, F.J. (2003): «El turismo cultural y el de sol y playa: ¿sustitutivos o complementarios?». Cuadernos de Turismo, $\mathrm{n}^{\circ} 11$, 97-105.

GETZ, D. (2008): «Event tourism: definition, evolution and research». Tourism Management, $\mathrm{n}^{\circ} 29$ (3), 403-428.

GONZALO, P. (2005): «El turismo cultural de Festivales y Eventos como herramienta para la segmentación de la demanda turística». Investigación y Marketing, $\mathrm{n}^{\circ}$ 87, 25-30.

HALL, C. M. (2007): Tourism planning. Policies, processes and relationships. (2 $2^{\mathrm{a}}$ ed.). Harlow, Prentice Hall.

IDESCAT (2012): «Dades estadístiques». Disponible en: http://www.idescat.cat.

INNERARITY, D. (2006): El nuevo espacio público. Madrid, Espasa Calpe.

KRUGMAN, P. (2010): La era de las expectativas limitadas. Barcelona, Ariel.

MINISTERIO DE CULTURA (2011): Encuesta de hábitos y prácticas culturales en España 2010-2011. Síntesis de resultados. Madrid, Ministerio de Cultura.

OBSERVATORI DEL TURISME DE CATALUNYA (2011): «Principals magnituds turístiques. Turisme a les marques catalanes». Barcelona, Departament d'Empresa i ocupación de la Generalitat de Catalunya.

PIRKKO, E.R. (2006): «Social Contacts and their Relationship to Loneliness among Aged People». Gerontology, n 52, 181-187.

ROMAGOSA, F., PRIESTLEY, G.K. y LLURDÉS, J.C. (2011): «El turismo en el marco de una estrategia de planificación sostenible general en Cataluña». Boletín de la Asociación de Geógrafos Españoles, n ${ }^{\circ}$ 7, 267-293.

RUHANEN, L. (2008): «Progressing the sustainability debate: A knowledge management approach to sustainable tourism planning». Current Issues in Tourism, $\mathrm{n}^{\circ} 11$ (5), 429-455.

SCOTT, J. (1991): Social Network Analysis. Newbury Park (Londres), Sage.

SHAPIRO, C. y VARIAN, H.R. (1999): The Information Rules: A Strategic Guide to the Network Economy. Harvard, Harvard Business School. 
SORENSEN, F. (2004): «Tourism Experience Innovation Networks». Tesis Doctoral. Universitat de Roskilde.

TABUENCA, M. (2002): «Festivales». Ier Congreso Internacional de Turismo Cultural. Disponible en: http: //www.turismo-cultural.org.

TKACZYNSKI A. y RUNDLE-THIELE, S.R. (2011): «Event segmentation: A review and research agenda ». Tourism Management, $\mathrm{n}^{\mathrm{o}} 32$, 426-434.

VERA, J.F. y BAÑOS, C.J. (2010): «Renovación y reestructuración de los destinos turísticos consolidados del litoral: las prácticas recreativas en la evolución del espacio turístico». Boletín de la Asociación de Geógrafos Españoles, n 53, 329-353.

WASSERMAN, N. y FAUST, K. (1994): Social Network Analysis: Methods and Applications. Carnbridge, University Press.

WELLMAN, B. (1999): Networks in the global village. Boulder, Westview Press.

WELlMAN, B. (2001): «Physical Place and Cyber-place: The rise of networked individualism». International Journal for Urban and Regional Research, $\mathrm{n}^{\circ}$ 25, 227-252.

WESTLUND, H. (1999): «An Interaction-Cost perspective on networks and territory». The Annals of Regional Science, $\mathrm{n}^{\circ} 33,93-121$. 
